\title{
Bethel basin, Alaska: 1979 field analyses sent to Doyon, Ltd.
}

Waller, S.F., and Amoco Oil Co.

GMC DATA REPORT 456

This GMC data report from the Amoco Heritage collection has been made available through funding from the FY2018 USGS National Geological and Geophysical Data Preservation Program, Grant Number G18AP00054. This project report is presented in its original format and has not been reviewed for technical content or for conformity to the editorial standards of DGGS. It should not be used or cited as reviewed data.

2019

State of Alaska

Department of Natural Resources

Division of Geological \& Geophysical Surveys
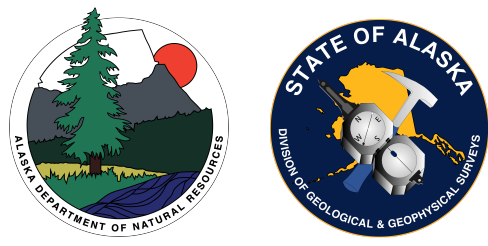


\section{CF820029}

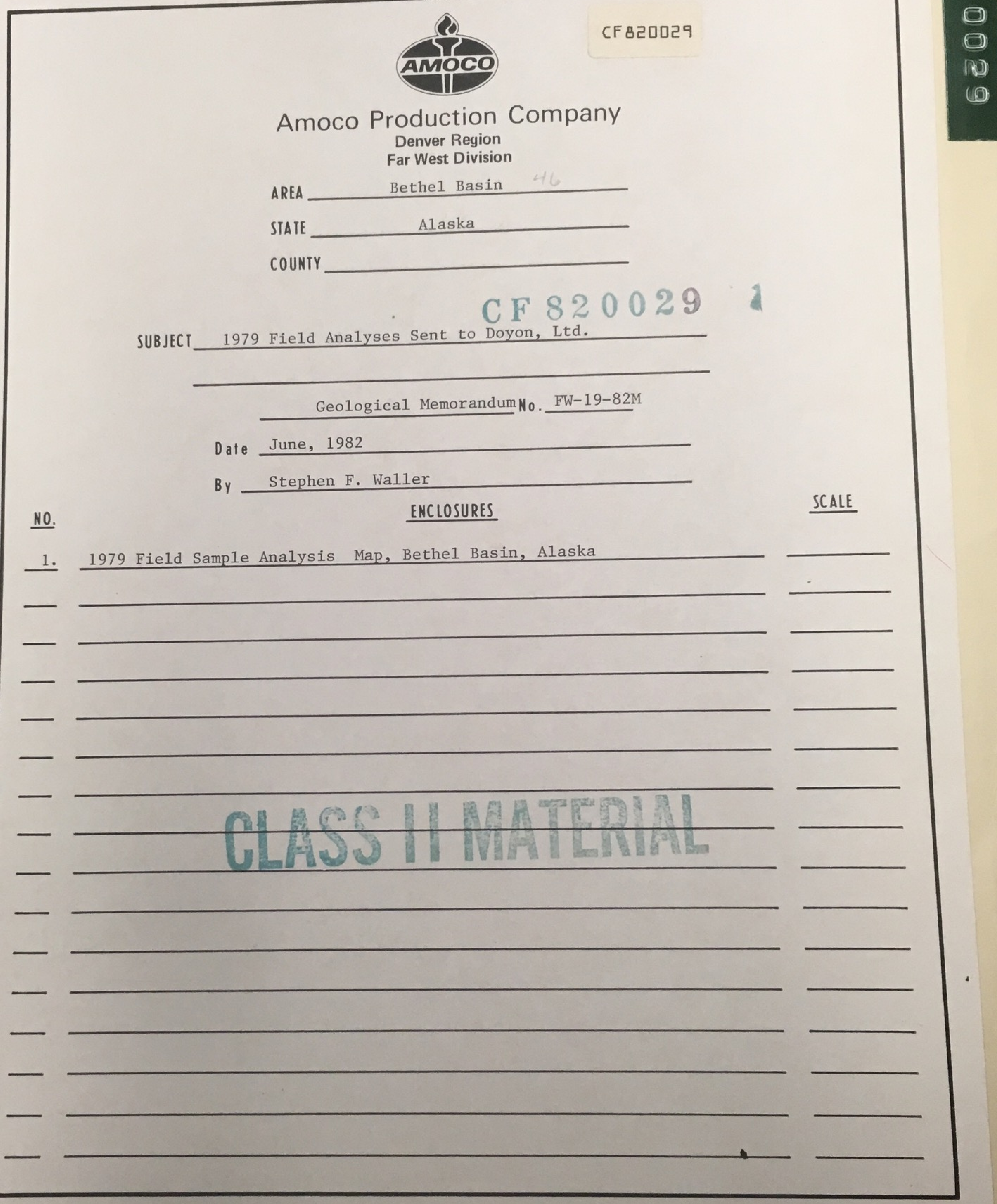




\section{Amoco Production Company}

Denver Region

Amoco Building

Denver, Colorado 80202

$303-830-4040$

June 10,1982

Doyon Ltd.

Doyon Bldg.

201 First Avenue

Fairbanks, Alaska 99701

Dear Sirs:

Enclosed please find the results of analyses of samples taken on Doyon Ltd. acreare by Mmoco's 1979 summer field narty (Bethel Basin/ florton Sound group) in July, 1979. This is all the sample data that is available, to the best of our knowledge. If additional analyses are ever performed, results will be provided to you upon completion.

Sincerely yours,

s\%

Stephen F. Waller

Geologist

SFW/cjp

CC: Scott McCoy 


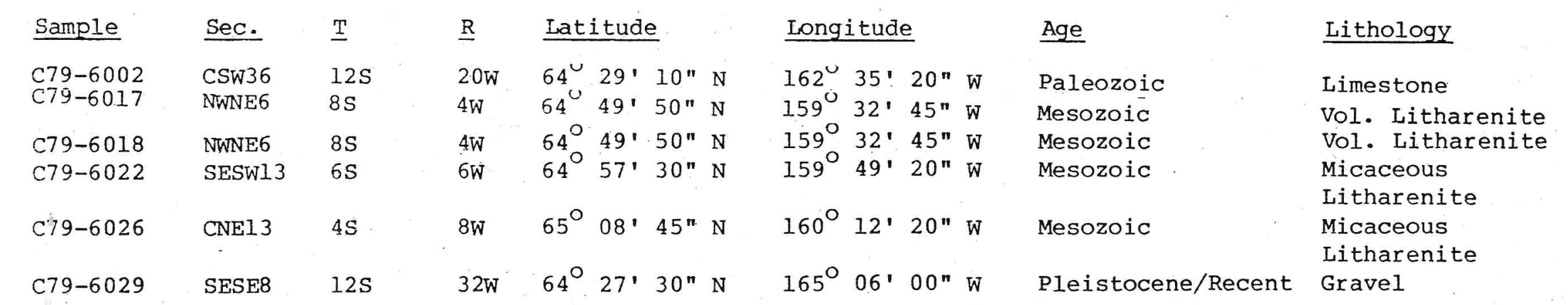

Petroleum

Generation

Capability

Kerogen

Stage of

Non-Source

Non-Source

Good

Fair

Non-Source (Gas)*

Non-Source
Type

$-$

Diagenesis

--
-
-

--
(Early Peak Gas) 


$\begin{array}{lll}\text { Sample } & \text { Sec. } & \underline{T} \\ \text { C79-4027 } & \text { SWNE3 } & 28 \text { S } \\ \text { C79-4028 } & \text { SWSW9 } & 28 \text { S } \\ \text { C79-4031 } & \text { NW19 } & 27 S \\ \text { C79-4032 } & \text { SESE10 } & 27 S \\ & & \\ \text { C79-4040 } & \text { SWSE33 } & 26 \mathrm{~S} \\ \text { C79-4042 } & \text { NE12 } & 22 \mathrm{~S} \\ \text { C79-4045 } & \text { SF.SF.34 } & 26 \mathrm{~S} \\ \text { C79-4048 } & \text { SESW21 } & 26 \mathrm{~S} \\ \text { C79-4075 } & \text { NENE9 } & 28 \mathrm{~S} \\ \text { C79-4077 } & \text { SWSW31 } & 27 S\end{array}$

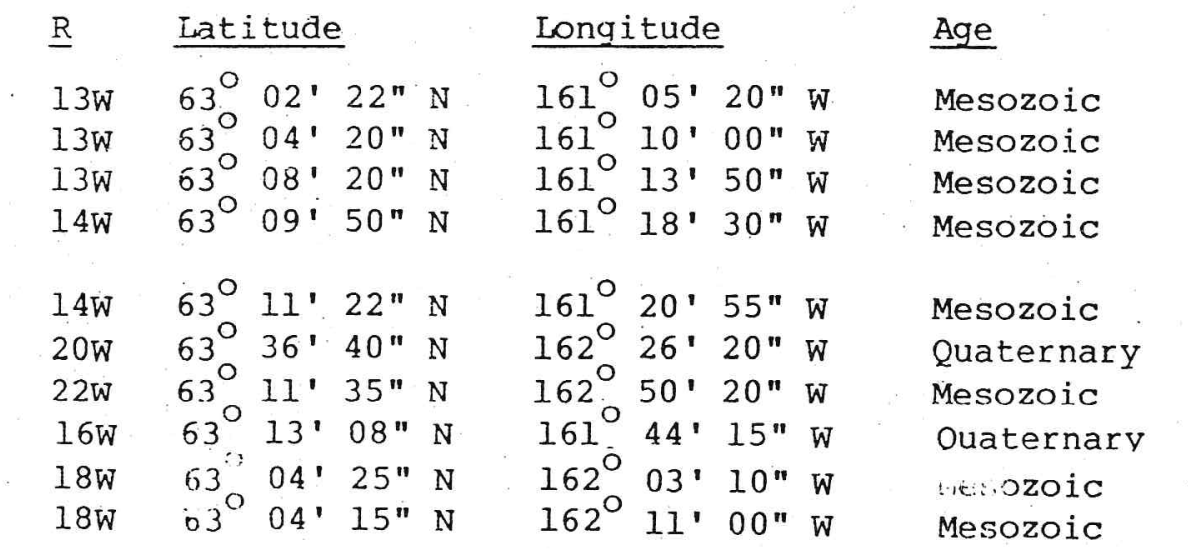

Lithology

Vol. SS

SS

(Micaceous)

SS/Siltstone

SS

Vesicular Basalt

Calc Litharenite

Basalt

SS

SS
Permeability

8 Porosity $(\varnothing)$

1.7

2.7

$<0.01$

0.01

$<0.01$

7.5

3.3

25.0

1.9

14.3

2.8

2.4
0.01

0.18

0.02

$<0.01$

$<0.01$ 


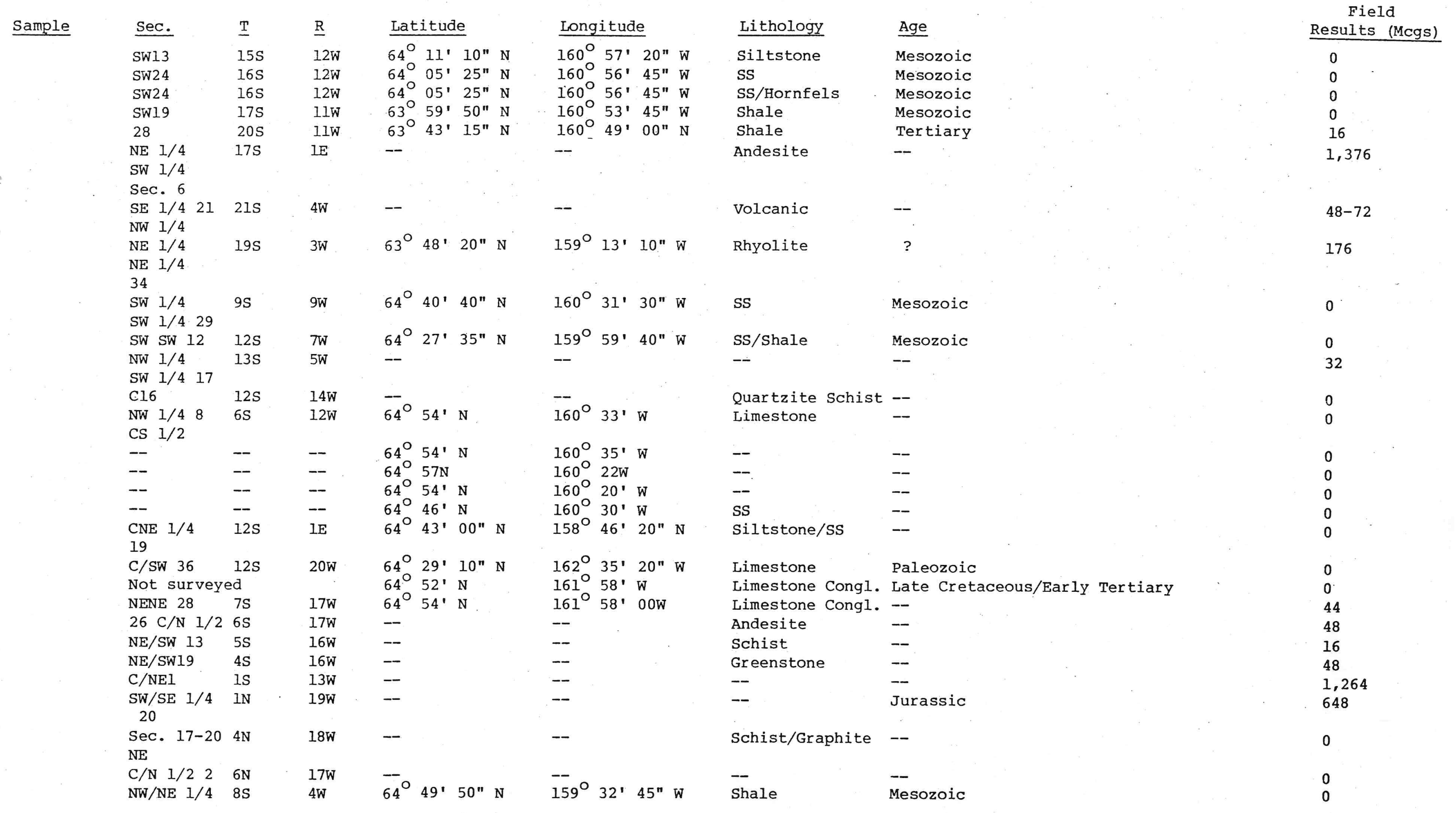




\begin{tabular}{|c|c|c|c|c|c|c|c|}
\hline Sample & Sec. & $\underline{T}$ & $\underline{\mathrm{R}}$ & \multicolumn{4}{|c|}{ Latitude } \\
\hline C79-4108 & NE8NW9 & $21 \mathrm{~s}$ & $9 \mathrm{~W}$ & $63^{\circ}$ & $41 '$ & $25 "$ & $\mathrm{~N}$ \\
\hline C79-4113 & SWSW6 & $22 \mathrm{~S}$ & $9 \mathrm{~W}$ & $63^{\circ}$ & $36^{\prime}$ & $30 "$ & $\mathrm{~N}$ \\
\hline C79-4116 & NWSE34 & $22 \mathrm{~s}$ & IOW & $63^{\circ}$ & $32^{\prime}$ & $20 "$ & $\mathrm{~N}$ \\
\hline C $79-4121$ & CW $1 / 2 \quad 15$ & $18 \mathrm{~S}$ & $5 \mathrm{~W}$ & $63^{\circ}$ & $55^{\prime}$ & $50 "$ & $\mathrm{~N}$ \\
\hline C79-4124 & NENE4 & $19 \mathrm{~S}$ & $6 \mathrm{~W}$ & $63^{\circ}$ & $52^{\prime}$ & $30 "$ & $\mathrm{~N}$ \\
\hline$C 79-4128$ & NENE28 & $19 \mathrm{~S}$ & $6 W$ & $63^{\circ}$ & $49^{\prime}$ & $15 "$ & $\mathrm{~N}$ \\
\hline C79-4129 & NWNW16 & $20 \mathrm{~S}$ & $6 \mathrm{~W}$ & $63^{\circ}$ & $45^{\prime}$ & $45^{\prime \prime}$ & $\mathrm{N}$ \\
\hline C $79-4132$ & NWSẆ3 & $22 \mathrm{~S}$ & $6 \mathrm{~W}$ & $63^{\circ}$ & $36^{\prime}$ & $35^{\prime \prime}$ & $\mathrm{N}$ \\
\hline$C 79-4135$ & SENEIO & $23 \mathrm{~S}$ & $7 \mathrm{~W}$ & $63^{\circ}$ & $45^{\prime}$ & $00 "$ & $\mathrm{~N}$ \\
\hline C79-4138 & CS $1 / 235$ & $22 \mathrm{~S}$ & $8 W$ & $63^{\circ}$ & $32^{\prime}$ & $30 "$ & $\mathrm{~N}$ \\
\hline C79-4139 & NWNW3 & $15 \mathrm{~s}$ & $1 \mathrm{E}$ & $64^{\circ}$ & $13^{\prime}$ & $30 "$ & $\mathrm{~N}$ \\
\hline C79-4144 & NWNENW17 & $22 \mathrm{~S}$ & $4 \mathrm{~W}$ & $63^{\circ}$ & $35^{\prime}$ & $20 "$ & $\mathrm{~N}$ \\
\hline$C 79-4148$ & SWSWIl & $19 \mathrm{~S}$ & $3 W$ & $63^{\circ}$ & $51^{\prime}$ & $15 "$ & $\mathrm{~N}$ \\
\hline C79-4151 & NWSE & $17 \mathrm{~S}$ & $4 \mathrm{~W}$ & $64^{\circ}$ & $01^{\prime}$ & $50 "$ & $\mathrm{~N}$ \\
\hline$C 79-4157$ & NENE34 & $16 \mathrm{~S}$ & 6w & $64^{\circ}$ & $03 !$ & $55^{\prime \prime}$ & $\mathrm{N}$ \\
\hline C 79-4165 & E $1 / 219$ & $17 \mathrm{~S}$ & $8 \mathrm{~W}$ & $64^{\circ}$ & $00^{\circ}$ & $25 "$ & $\mathrm{~N}$ \\
\hline & $\begin{array}{lll}W & 1 / 2 & 20\end{array}$ & & & & & & \\
\hline$C 79-4168$ & NWNW9 & $16 \mathrm{~S}$ & $9 \mathrm{~W}$ & $64^{\circ}$ & $07^{\prime}$ & $15 "$ & $\mathrm{~N}$ \\
\hline C79-4172 & NENE18 & $15 \mathrm{~S}$ & $9 \mathrm{~W}$. & $64^{\circ}$ & $11{ }^{\prime}$ & $35 "$ & $\mathrm{~N}$ \\
\hline C79-4178 & NWNW8 & $13 \mathrm{~s}$ & $11 \mathrm{~W}$ & $64^{\circ}$ & $22^{\prime}$ & $50 "$ & $\mathrm{~N}$ \\
\hline C79-4182 & SWSW21 & $11 \mathrm{~s}$ & 10w & $64^{\circ}$ & $30^{\prime}$ & $50 "$ & $\mathrm{~N}$ \\
\hline C79-4185 (a) & NESW33 & $9 \mathrm{~S}$ & $9 \mathrm{~W}$ & $64^{\circ}$ & $24^{\prime}$ & $00 "$ & $\mathrm{~N}$ \\
\hline C79-4185 (b) & NESW33 & 9S. & $9 \mathrm{~W}$ & $64^{\circ}$ & $24^{\prime}$ & $00 "$ & $\mathrm{~N}$ \\
\hline C79-4190 (a) & SESW5 & 115 & $8 \mathrm{~W}$ & $64^{\circ}$ & $33^{\prime}$ & $40 "$ & $\mathrm{~N}$ \\
\hline C79-4193(a) & NWSW25 & $11 \mathrm{~s}$ & $8 \mathrm{~W}$ & $64^{\circ}$ & $30^{\prime}$ & $25 "$ & $\mathrm{~N}$ \\
\hline C79-4198 & SWSW29 & $12 \mathrm{~S}$ & $6 \mathrm{~W}$ & $64^{\circ}$ & $25^{\prime}$ & $08 "$ & $\mathrm{~N}$ \\
\hline C79-4201 & NWSW17 & $13 \mathrm{~S}$ & $5 \mathrm{~W}$ & $64^{\circ}$ & $21^{\prime}$ & $50 "$ & $\mathrm{~N}$ \\
\hline C79-4205 & NENE26 & $14 \mathrm{~S}$ & $5 \mathrm{~W}$ & $64^{\circ}$ & $15^{\prime}$ & $25 "$ & $\mathrm{~N}$ \\
\hline C79-4208 & SWNW14 & $15 \mathrm{~S}$ & $4 W$ & $64^{\circ}$ & $11^{\prime}$ & $35 "$ & $\mathrm{~N}$ \\
\hline C79-4215 & NE8 & 135 & $14 \mathrm{~W}$ & $64^{\circ}$ & $22^{\prime}$ & $25^{\prime \prime}$ & $\mathrm{N}$ \\
\hline C $79-4229$ & NWSE13 & $9 \mathrm{~S}$ & $1 W$ & $64^{\circ}$ & $43^{\prime}$ & $00 "$ & $\mathrm{~N}$ \\
\hline$C 79-4232$ & Not surve & & & $64^{\circ}$ & $46^{\prime}$ & $50 "$ & $\mathrm{~N}$ \\
\hline C79-4235 & SESE34 & $7 \mathrm{~S}$ & $2 W$ & $64^{\circ}$ & $50^{\prime}$ & $00 "$ & $\mathrm{~N}$ \\
\hline C $79-4155$ & SWNW13 & $16 \mathrm{~S}$ & $5 \mathrm{~W}$ & $64^{\circ}$ & $06^{\prime}$ & $15 "$ & $\mathrm{~N}$ \\
\hline
\end{tabular}

\section{Long i tude}

$160^{\circ} 26^{\prime} 00^{\prime \prime} \mathrm{W}$ $160^{\circ} 29^{\prime} 40^{\prime \prime} \mathrm{W}$ $160^{\circ} 34$ ' 45" W $159^{\circ} 49^{\prime} 45^{\prime \prime} \mathrm{W}$ $159^{\circ} 49^{\prime} 10^{\prime \prime} \mathrm{W}$ $159^{\circ} 50^{\prime} 00^{\prime \prime} \mathrm{W}$ $159^{\circ} 51$ ' $30^{\prime \prime} \mathrm{W}$ $159^{\circ} 48^{\prime} 50^{\prime \prime} \mathrm{W}$ $159^{\circ} 59^{\prime} 30^{\prime \prime} \mathrm{W}$ $160^{\circ} 09^{\prime} 00^{\prime \prime} \mathrm{W}$ $158^{\circ} 39^{\prime} 25^{\prime \prime} \mathrm{W}$ $159^{\circ} 29^{\prime} 32^{\prime \prime} \mathrm{W}$ $159^{\circ} 12^{\prime} 50^{\prime \prime} \mathrm{W}$ $159^{\circ} 27^{\prime} 25^{\prime \prime} \mathrm{W}$ $159^{\circ} 49^{\prime} 00^{\prime \prime} \mathrm{W}$ $160^{\circ} 17^{\prime} 10^{\prime \prime} \mathrm{W}$

$160^{\circ} 27^{\prime} 45^{\prime \prime} \mathrm{W}$ $160^{\circ} 30^{\prime} 45^{\prime \prime} \mathrm{W}$ $160^{\circ} 47^{\prime} 40^{\prime \prime} \mathrm{W}$ $160^{\circ} 34^{\prime} 35^{\prime \prime} \mathrm{W}$ $160^{\circ} 29^{\prime} 00^{\prime \prime} \mathrm{W}$ $160^{\circ} 29^{\prime} 00^{\prime \prime} \mathrm{W}$ $160^{\circ} 29,10^{\prime \prime} W$ $160^{\circ} 20^{\prime}, 11^{\prime \prime} \mathrm{W}$ $159^{\circ} 55^{\prime} 15^{\prime \prime} \mathrm{W}$ $159^{\circ} 42^{\prime} 25^{\prime \prime} \mathrm{W}$ $159^{\circ} 35^{\prime} 25^{\prime \prime} \mathrm{W}$ $159^{\circ} 23^{\prime} 20^{\prime \prime} \mathrm{W}$ $161^{\circ} 30^{\prime} 50^{\prime \prime} \mathrm{W}$ $158^{\circ} 46^{\prime} 20^{\prime \prime} \mathrm{W}$ $158^{\circ} 49^{\prime} 50^{\prime \prime} \mathrm{W}$ $159^{\circ} 02^{\prime} 00^{\prime \prime} \mathrm{W}$ $159^{\circ} 34^{\prime} 30^{\prime \prime} \mathrm{W}$
Lithology Age

Field

Results (Mcgs)

Mesozoic

Shaley Siltstone Mesozoic Mesozoic Shaley Siltstone Mesozoic SS Mesozoic Silty Shale Mesozoic Silty Shale Mesozoic SS SS ?

Silty Shale

Siltstone

SS

$\begin{array}{ll}\text { SS } & \text { Mesozoic } \\ \text { SS } & \text { Mesozoic }\end{array}$

Mesozoic

?

$?$

$?$

Mesozoic Mesozoic Mesozoic Mesozoic

Mesozoic

Mesozoic Mesozoic Mesozoic Mesozoic Mesozoic Mesozoic Mesozoic Mesozoic Mesozoic Mesozoic Mesozoic Mesozoic

120

28

40

32

40

.2


Magnetic Susceptibility

\begin{tabular}{|c|c|c|c|c|c|c|c|c|c|c|c|c|}
\hline Sample & Sec. & $\underline{T}$ & $\underline{\mathrm{R}}$ & Lati & itude & & & Long & tude & & & Age \\
\hline C79-4018 & 28 & $20 \mathrm{~s}$ & IIW & $63^{\circ}$ & $43^{\prime}$ & $15^{\prime \prime}$ & $\mathrm{N}$ & $160^{\circ}$ & $49^{\prime}$ & $0.0 "$ & W & Shale \\
\hline C $79-4027$ & STNE3 & $28 \mathrm{~S}$ & $13 \mathrm{~W}$ & $63^{\circ}$ & $02^{\prime}$ & $22^{n}$ & $\mathrm{~N}$ & $161^{\circ}$ & $05^{\prime}$ & $20^{\prime \prime}$ & W & Volcanic SS \\
\hline C79-4028 & SWSW9 & $28 \mathrm{~S}$ & $13 \mathrm{~W}$ & $63^{\circ}$ & $04^{\prime}$ & $20^{\prime \prime}$ & $\mathrm{N}$ & $161^{\circ}$ & $10^{\prime}$ & $00 "$ & W & ss \\
\hline C79-4031 & NW19 & $27 \mathrm{~S}$ & $13 \mathrm{~W}$ & $63^{\circ}$ & $08^{\prime}$ & $20 "$ & $\mathrm{~N}$ & $161^{\circ}$ & $13^{\prime}$ & $50 "$ & $\mathrm{w}$ & SS \\
\hline C79-4032 & SESEIO & $27 \mathrm{~S}$ & $14 \mathrm{~W}$ & $63^{\circ}$ & $09^{\prime}$ & $50 "$ & $\mathrm{~N}$ & $161^{\circ}$ & $18^{\prime}$ & $30 "$ & $\mathrm{~W}$ & ss/siltstone \\
\hline C79-4040 & SWSE33 & $26 \mathrm{~S}$ & $14 \mathrm{~W}$ & $63^{\circ}$ & $11{ }^{\prime}$ & $22 "$ & $\mathrm{~N}$ & $161^{\circ}$ & $20^{\prime}$ & $55^{\prime \prime}$ & w & SS \\
\hline C $79-4042$ & NEl2 & 225 & $20 \mathrm{~W}$ & $63^{\circ}$ & $36^{\prime}$ & $40^{\prime \prime}$ & $\mathrm{N}$ & $162^{\circ}$ & $26^{\prime}$ & $20 "$ & W & Basalt \\
\hline C79-4048 & SESW21 & $26 \mathrm{~s}$ & $16 \mathrm{~W}$ & $63^{\circ}$ & $13^{\prime}$ & $00 !$ & $\mathrm{N}$ & $161_{0}^{\circ}$ & $44^{\prime}$ & $15^{n}$ & $\mathrm{w}$ & Basalt \\
\hline C $79-4084$ & SESEIO & $20 \mathrm{~S}$ & $10 \mathrm{~W}$ & $63^{\circ}$ & $46^{\prime}$ & $00 "$ & $\mathrm{~N}$ & $160^{\circ}$ & $35^{\prime}$ & $00 "$ & W & Shale \\
\hline C79-4091 & NWNE30 & $21 \mathrm{~s}$ & 10W & $63^{\circ}$ & $38^{\prime}$ & $45 "$ & $\mathrm{~N}$ & $160^{\circ}$ & $40^{\prime}$ & $30 "$ & w & Shale \\
\hline$C 79-4093$ & $\mathrm{~S} \quad 1 / 2 \quad 13$ & 225 & $11 W$ & $63^{\circ}$ & $34^{\prime}$ & $30 "$ & $\mathrm{~N}$ & $160^{\circ}$ & $42^{\prime}$ & $50 "$ & W & SS \\
\hline & N. $1 / 224$ & & & & & & & & & & & \\
\hline C79-4095 & CNE15 & $23 \mathrm{~S}$ & $11 \mathrm{~W}$ & $63^{\circ}$ & $30^{\prime}$ & $00^{\prime \prime}$ & $\mathrm{N}$ & $160^{\circ}$ & $45^{\prime}$ & $50 "$ & $\mathrm{~W}$ & SS \\
\hline C79-4099 & SWSW19 & $23 \mathrm{~S}$ & $11 \mathrm{~W}$ & $63^{\circ}$ & $28^{\prime}$ & $40 "$ & $\mathrm{~N}$ & $160^{\circ}$ & $52^{\prime}$ & $45^{\prime \prime}$ & W & SS \\
\hline C79-4104 & NESE2 2 & $23 \mathrm{~S}$ & $12 \mathrm{~W}$ & $63^{\circ}$ & $28^{\prime}$ & $55^{\prime \prime}$ & $\mathrm{N}$ & $160^{\circ}$ & $56^{\prime}$ & $50^{n}$ & $\mathrm{w}$ & SS \\
\hline
\end{tabular}

Lithology

Tertiary

Mesozoic

Mesozoic

Mesozoic

Mesozoic

Mesozoic

Quaternary

Quaternary

Mesozoic

Mesozoic

Mesozoic

Mesozoic

Mesozoic

Mesozoic
Field

Results

Results (Mcgs)

(Mcgs)
$-$

76

600

48

$32-60$

40.

36

36

48

40

60

32 


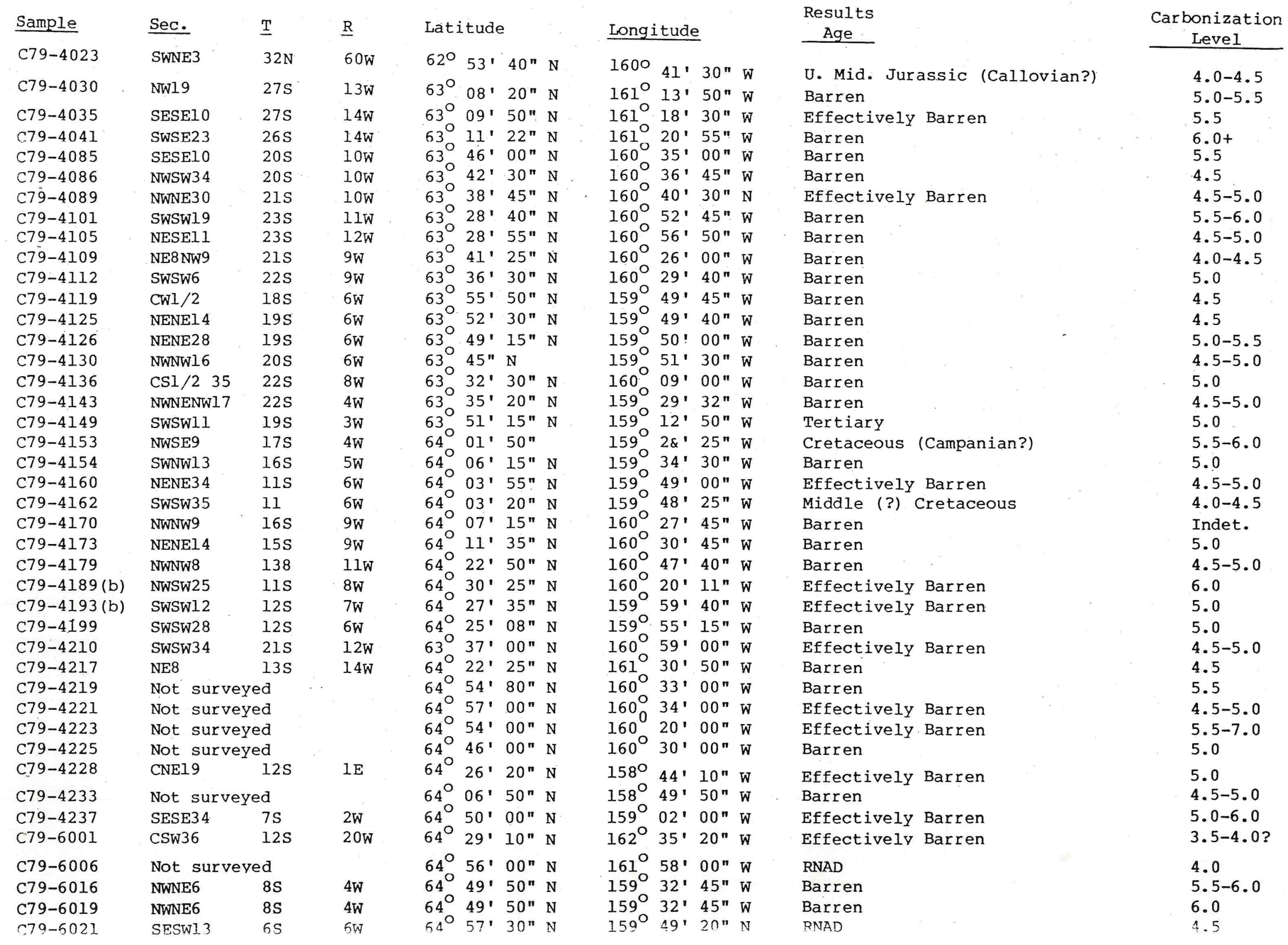




\begin{tabular}{|c|c|c|c|c|c|c|c|c|c|c|c|c|c|}
\hline Sample & $\underline{\text { Sec. }}$ & $\underline{T}$ & $\underline{\mathrm{R}}$ & Lat : & itude & & & Long i & itude & & & $\begin{array}{l}\text { Results: } \\
\text { Age } \\
\end{array}$ & Lithology \\
\hline C79-2003 & NW16 & $6 \mathrm{~S}$ & $69 \mathrm{~W}$ & $59^{\circ}$ & $39^{\prime}$ & $40 "$ & $\mathrm{~N}$ & $161^{\circ}$ & 02 ' & $00 "$ & W & $104 \div 4 \mathrm{my}$ & Vesicular Basalt \\
\hline C79-2005 & NENEI 9 & $5 S$ & $68 \mathrm{~W}$ & $59^{\circ}$ & $44^{\prime}$ & $20^{n}$ & $\mathrm{~N}$ & $160^{\circ}$ & $5.4^{\prime}$ & $25 "$ & $w$ & $103 \pm 4 m y$ & Andesite \& Breccia \\
\hline C79-2029 & SWNE8 & $2 \mathrm{~N}$ & $66 \mathrm{~W}$ & $60^{\circ}$ & $14^{\prime}$ & $10^{n}$ & $\mathrm{~N}$ & $160^{\circ}$ & $46^{\prime}$ & $30^{\prime \prime}$ & $W$ & $57.0 \pm 2.7 \mathrm{my}$ & Rhyolite \\
\hline C79-2031 & NWSE30 & $3 \mathrm{~N}$ & $66 \mathrm{~W}$ & $60^{\circ}$ & $19^{\prime}$ & $12 "$ & $\mathrm{~N}$ & $160^{\circ}$ & $48^{\prime}$ & $00^{n}$ & $\mathrm{~W}$ & $66.0 \mp 2.8 \mathrm{my}$ & Basalt \\
\hline C79-2041 & NENE2 1 & $3 \mathrm{~N}$ & $66 \mathrm{~W}$ & $60^{\circ}$ & $20^{\prime}$ & $40^{\prime \prime}$ & $\mathrm{N}$ & $160^{\circ}$ & $44^{\prime}$ & $30^{n}$ & $\mathrm{~W}$ & $60.4 \mp 2.5 \mathrm{my}$ & Rhyolite \\
\hline C79-2042 & NENE2 1 & $3 \mathrm{~N}$ & $66 \mathrm{~W}$ & $60^{\circ}$ & $20^{\prime}$ & $40^{n}$ & $\mathrm{~N}$ & $160^{\circ}$ & $44^{\prime}$ & $30 "$ & W & $62.8 \pm 2.8 \mathrm{my}$ & Basalt \\
\hline C79-2064 & SENW30 & $6 \mathrm{~N}$ & $6.5 W$ & $60^{\circ}$ & $34^{\prime}$ & $40^{\prime \prime}$ & $\mathrm{N}$ & $160^{\circ}$ & $45^{\prime}$ & $20 "$ & $\mathrm{~W}$ & $112 \pm 5 \mathrm{my}$ & Basalt \\
\hline C79-2066 & NWNE6 & $5 \mathrm{~N}$ & $65 \mathrm{~W}$ & $60_{0}^{\circ}$ & $33^{\prime}$ & $40 "$ & $\mathbf{N}$ & $160^{\circ}$ & $45^{\prime}$ & $20^{\prime \prime}$ & $\mathrm{W}$ & $114 \pm 6 \mathrm{my}$ & Basalt Vesicular \\
\hline$C 79-2067$ & NWSE3 & $5 \mathrm{~N}$ & $65 w$ & $60^{\circ}$ & $33^{\prime}$ & $10^{n}$ & $\mathrm{~N}$ & $160_{0}^{\circ}$ & $39^{\prime}$ & $58 "$ & $\mathrm{~W}$ & $127 \pm+6$ miy & Basalt Pillows \\
\hline C79-2069 & NWNE2 4 & $5 \mathrm{~N}$ & $65 \mathrm{~W}$ & $60^{\circ}$ & $30^{\prime}$ & $50 "$ & $\mathrm{~N}$ & $160^{\circ}$ & $36^{\prime}$ & $20^{\prime \prime}$ & $\mathrm{W}$ & $63.5 \mp 2.8 \mathrm{my}$ & Basalt \& Volcaniclastics \\
\hline C79-2084 & NWNW33 & $6 \mathrm{~N}$ & $64 \mathrm{~W}$ & $60^{\circ}$ & $34^{\prime}$ & $15 "$ & $\mathrm{~N}$ & $160^{\circ}$ & $30^{\prime}$ & $15^{n}$ & $\mathrm{~W}$ & $61.3 \frac{ \pm}{\mp} 2.7 \mathrm{my}$ & Basalt/Diorite \\
\hline C79-2086 & NESW27 & $6 \mathrm{~N}$ & $64 \mathrm{~W}$ & $60^{\circ}$ & $34^{\prime}$ & $47 "$ & $\mathrm{~N}$ & $160^{\circ}$ & $29^{\prime}$ & $58^{n}$ & $\mathrm{~W}$ & $67.3 \pm 2.7 \mathrm{my}$ & Rhyolite \\
\hline C79-2089 & NWSE2I & $7 \mathrm{~N}$ & $65 \mathrm{~W}$ & $60^{\circ}$ & $40^{\prime}$ & $45 "$ & $\mathrm{~N}$ & $160^{\circ}$ & $41^{\prime}$ & $45^{\prime}$ & $\mathrm{W}$ & $127 \pm 7 \mathrm{my}$ & Andesite \\
\hline C79-2093 & SwSw34 & $7 \mathrm{~N}$ & $63 w$ & $60^{\circ}$ & $39^{\prime}$ & $10 "$ & $\mathrm{~N}$ & $160^{\circ}$ & $19^{\prime}$ & $15^{n}$ & W & $53.0 \pm 4.0 \mathrm{my}$ & Basalt \\
\hline C79-2125 & NWNE2 8 & $5 \mathrm{~N}$ & $60 \mathrm{~W}$ & $60^{\circ}$ & $30^{\prime}$ & $50 "$ & $\mathrm{~N}$ & $159^{\circ}$ & $48^{\prime}$ & $40 "$ & $\mathrm{~W}$ & $127-16 \mathrm{my}$ & Basalt \\
\hline C79-2143 & Not sur & & & $60^{\circ}$ & $22^{\prime}$ & $\mathrm{N}$ & & $166^{\circ}$ & $24^{\prime}$ & $\mathrm{W}$ & & $47.0 \pm 2.1 \mathrm{my}$ & Basalt \\
\hline C79-2148 & NWSW18 & $15 \mathrm{~N}$ & $82 \mathrm{~W}$ & $61^{\circ}$ & $23^{\prime}$ & $15 "$ & $\mathrm{~N}$ & $164^{\circ}$ & $04^{\prime}$ & $10 "$ & $\mathrm{~W}$ & $0.71 \mp .07 \mathrm{my}$ & Basalt \\
\hline C79-2156 & NESW23 & $7 \mathrm{~N}$ & $90 \mathrm{~W}$ & $60^{\circ}$ & $40^{\prime}$ & $50^{\prime \prime}$ & $\mathrm{N}$ & $165^{\circ}$ & $0 I^{\prime}$ & $50^{n}$ & $\mathrm{~W}$ & $11.9 \div 1.3 \mathrm{my}$ & Volcanic \\
\hline C79-2157 & NWNW10 & $22 \mathrm{~N}$ & $83 W$ & $62^{\circ}$ & $00^{\prime}$ & $55^{\prime \prime}$ & $\mathrm{N}$ & $164^{\circ}$ & $28^{\prime}$ & $36^{n}$ & $\mathrm{~W}$ & $59.9 \pm 2.3 \mathrm{my}$ & Lamprophyre \\
\hline C79-2161 & SENW20 & $24 \mathrm{~N}$ & $81 \mathrm{~W}$ & $62^{\circ}$ & $09^{\prime}$ & $32 "$ & $\mathrm{~N}$ & $164^{\circ}$ & $10^{\prime}$ & $25^{n}$ & $\mathrm{~W}$ & $4.0 \pm .4 \mathrm{my}$ & Basalt \\
\hline C79-2163 & Not sur & & & $62^{\circ}$ & $28^{\prime}$ & $55 "$ & $\mathrm{~N}$ & 164 & $31^{\prime}$ & $15^{n}$ & $\mathrm{~W}$ & $7.1 \pm .6 \mathrm{my}$ & Basalt \\
\hline C79-2206 & SESEl I & $10 \mathrm{~N}$ & $58 \mathrm{~W}$ & $60^{\circ}$ & $59^{\prime}$ & $10^{n}$ & $\mathrm{~N}$ & $159^{\circ}$ & $43^{\prime}$ & $30^{n}$ & $\mathrm{~W}$ & $71.6 \mp 2.7 \mathrm{my}$ & Granodiorite \\
\hline C79-2208 & NWSWII & $18 \mathrm{~N}$ & $55 \mathrm{~W}$ & $61^{\circ}$ & $39^{\prime}$ & $45^{\prime \prime}$ & $\mathrm{N}$ & $159^{\circ}$ & $13^{\prime}$ & $00^{n}$ & $\mathrm{~W}$ & $73.6 \pm 2.7 \mathrm{my}$ & Syenite \\
\hline C79-2218 & Not sur & & & $62^{\circ}$ & $45^{\prime}$ & $00^{\prime \prime}$ & $\mathrm{N}$ & $163^{\circ}$ & $32^{\prime}$ & $32^{n}$ & w & $97.2 \mp 3.6 \mathrm{my}$ & Basalt \& Breccia \\
\hline C79-2246 & NWNW3 & $30 \mathrm{~N}$ & $68 \mathrm{~W}$ & $62 \circ$ & $43^{\prime}$ & $25 "$ & $\mathrm{~N}$ & $162^{\circ}$ & $01^{\prime}$ & $10^{n}$ & W & $56.3 \mp 2.4 \mathrm{my}$ & Rhyolite \\
\hline C79-2250 & NWNW10 & $18 \mathrm{~N}$ & $6 \mathrm{~W}$ & $61^{\circ}$ & $40^{\prime}$ & $15^{\prime \prime}$ & $\mathrm{N}$ & $160^{\circ}$ & $08^{\prime}$ & $30^{n}$ & $\mathrm{w}$ & $83.8 \div 3.2 \mathrm{my}$ & Andesite \\
\hline C79-2276 & NWSE9 & $21 \mathrm{~N}$ & $64 \mathrm{~W}$ & $61_{0}^{\circ}$ & $55^{\prime}$ & $20^{n}$ & $\mathrm{~N}$ & $161_{0}^{\circ}$ & $011^{\prime}$ & $10^{n}$ & W & $56.6 \pm 2.3 \mathrm{my}$ & Andesite Breccia \\
\hline C79-2281 & SWSW17 & $26 \mathrm{~N}$ & $66 \mathrm{~W}$ & $62^{\circ}$ & $20^{\prime}$ & $15 "$ & $\mathrm{~N}$ & $161^{\circ}$ & $34^{\prime}$ & $10^{n}$ & W & $61.6 \pm 2 \mathrm{my}$ & Basalt \\
\hline C79-2283 & NESW13 & $26 \mathrm{~N}$ & $66 \mathrm{~W}$ & $62^{\circ}$ & $20^{\prime}$ & $55^{n}$ & $\mathrm{~N}$ & $161_{0}^{\circ}$ & $26^{\prime}$ & $20^{n}$ & $W$ & No daţe & Dacite \\
\hline C79-2288 & SENEl 8 & $26 \mathrm{~N}$ & $61 \mathrm{~W}$ & $62^{\circ}$ & $20^{\prime}$ & $45^{\prime \prime}$ & $\mathrm{N}$ & $160^{\circ}$ & $39^{\prime}$ & $00^{n}$ & W & $52.1 \pm 2 \mathrm{my}$ & Andesite \\
\hline $\begin{array}{l}C 79-6007 \\
C 79-6009\end{array}$ & $\begin{array}{l}\text { CNE27 } \\
\text { SWNW15 }\end{array}$ & $\begin{array}{l}5 \mathrm{~S} \\
2 \mathrm{~N}\end{array}$ & $\begin{array}{l}17 \mathrm{~W} \\
19 \mathrm{~W}\end{array}$ & $65^{\circ}$ & $\begin{array}{l}06^{\prime} \\
34,\end{array}$ & $\begin{array}{l}30 n \\
40^{n}\end{array}$ & $\begin{array}{l}\mathrm{N} \\
\mathrm{N}\end{array}$ & $\begin{array}{l}162^{\circ} \\
160^{\circ}\end{array}$ & $\begin{array}{l}04{ }^{\prime} \\
34 !\end{array}$ & $\begin{array}{l}35^{n} \\
15 n\end{array}$ & $W$ & $151 \pm 10 \mathrm{my}$ & $\begin{array}{l}\text { Andesite } \\
\text { Basalt }\end{array}$ \\
\hline C79-6010 & $\begin{array}{l}\text { SWNW15 } \\
\text { SENW2 }\end{array}$ & $\begin{array}{l}2 \mathrm{~N} \\
1 \mathrm{~N}\end{array}$ & $\begin{array}{l}19 \mathrm{~W} \\
24 \mathrm{~W}\end{array}$ & $65^{\circ}$ & 31. & $12^{n}$ & $\mathrm{~N}$ & $163^{\circ}$ & $36^{\prime}$ & $00^{n}$ & W & $\begin{array}{l}34.8 \pm 2.1 \mathrm{my} \\
2.1 \pm 1.2 \mathrm{my}\end{array}$ & $\begin{array}{l}\text { Basalt } \\
\text { Basalt }\end{array}$ \\
\hline C79-6030 & NWNW36 & 115 & $38 w$ & $64^{\circ}$ & $29^{\prime}$ & $30^{n}$ & $\mathrm{~N}$ & $166^{\circ}$ & $12^{\prime}$ & $20^{n}$ & $\mathrm{~W}$ & $104 \pm 4 \mathrm{my}$ & Granite \\
\hline$C 79-6047$ & Not sur & & & $64^{\circ}$ & $58^{\prime}$ & $00^{n}$ & $\mathrm{~N}$ & $168^{\circ}$ & $02^{\prime}$ & $00^{n}$ & W & $103 \pm 4 \mathrm{my}$ & Granite \\
\hline C79-6064 & NWNW15 & $6 \mathrm{~N}$ & $36 \mathrm{~W}$ & $65^{\circ}$ & $55^{\prime}$ & $30^{n}$ & $\mathrm{~N}$ & $166^{\circ}$ & $14^{\prime}$ & $25 "$ & $W$ & $71.2 \pm 2.6 \mathrm{my}$ & Granite \\
\hline C79-6066 & NWNElI & $4 \mathrm{~S}$ & $37 \mathrm{~W}$ & $65^{\circ}$ & $09^{\prime}$ & $45 "$ & $\mathrm{~N}$ & $166^{\circ}$ & $11^{\prime}$ & $50^{\prime \prime}$ & $\mathrm{W}$ & $388 \pm 28 \mathrm{my}$ & Granite \\
\hline
\end{tabular}


Density

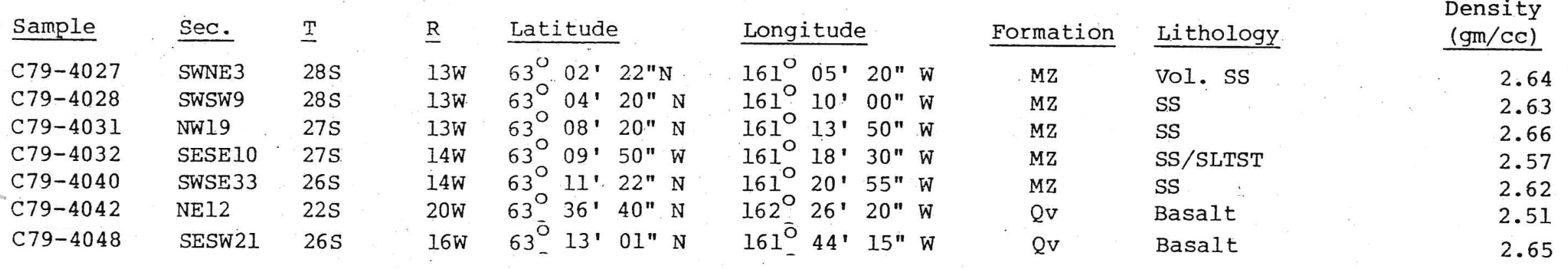

